\title{
Preoperative serum sodium measurements and postoperative inpatient mortality: A case-control analysis of data from the South African Surgical Outcomes Study
}

\author{
M Ramburuth, ${ }^{1,2} \mathrm{MB} \mathrm{ChB}, \mathrm{DA}(\mathrm{SA}) ;$ Y Moodley, ${ }^{1} \mathrm{PhD} ; \mathbf{P}$ D Gopalan, ${ }^{1} \mathrm{MB}$ ChB, FCA (SA), Crit Care (SA) \\ ${ }^{1}$ Discipline of Anaesthesiology and Critical Care Medicine, Nelson R Mandela School of Medicine, College of Health Sciences, \\ University of KwaZulu-Natal, Durban, South Africa \\ ${ }^{2}$ Inkosi Albert Luthuli Central Hospital, Durban, South Africa
}

Corresponding author: Y Moodley (moodleyyo@ukzn.ac.za)

Background. Abnormal preoperative serum sodium measurements have been shown to be associated with increased postoperative mortality in US and European surgical populations. It is possible that such measurements are also associated with increased postoperative mortality in a South African (SA) setting, but this is yet to be confirmed. Establishing whether preoperative serum sodium measurements are associated with postoperative mortality could have implications for perioperative risk stratification in SA settings.

Objectives. To determine whether preoperative serum sodium measurements are associated with postoperative mortality in SA surgical patients.

Methods. This was an unmatched case-control study of patient data (demographics, comorbidities, procedure-related variables, and preoperative serum sodium measurements) collected during the South African Surgical Outcomes Study. Data were analysed using recommended statistical methods for unmatched case-control studies.

Results. The study population comprised 103 patients and 410 controls. Cases were defined as patients who suffered postoperative inpatient mortality, while controls were defined as patients who did not suffer postoperative inpatient mortality. Preoperative hypernatraemia (i.e. a preoperative serum sodium measurement $>144 \mathrm{mEq} / \mathrm{L}$ ) was independently associated with a four-fold higher risk of postoperative inpatient mortality compared with a normal preoperative serum sodium measurement of $135-144 \mathrm{mEq} / \mathrm{L}$ (odds ratio (OR) 4.21 , 95\% confidence interval (CI) 1.19 - 14.83, $p=0.025$ ). Preoperative hyponatraemia (i.e. a preoperative serum sodium measurement $<135 \mathrm{mEq} / \mathrm{L}$ ) was not independently associated with a higher or lower risk of postoperative inpatient mortality compared with a normal preoperative serum sodium measurement (OR 1.39, 95\% CI $0.70-2.76, p=0.346$ ).

Conclusions. Preoperative hypernatraemia, but not preoperative hyponatraemia, is a risk factor for postoperative inpatient mortality in SA surgical patients.

S Afr Med J 2018;108(10):847-851. DOI:10.7196/SAMJ.2018.v108i10.13178

Sodium measurements are often performed during the preoperative anaesthetic workup in surgical patients. ${ }^{[1]}$ Preoperative dysnatraemia (a preoperative serum sodium measurement outside the normal range of 135 - $144 \mathrm{mEq} / \mathrm{L}$ ) is a common electrolyte imbalance in noncardiac surgical patients. ${ }^{[2,3]}$ An estimated $40 \%$ of non-cardiac surgical patients in the European Surgical Outcomes Study (EuSOS) were reported to have had an abnormal preoperative sodium measurement. ${ }^{[1]}$

The clinical relevance of preoperative dysnatraemia is gaining acknowledgement. A study by $\mathrm{Hu}$ et al. ${ }^{[4]}$ suggests that not only is dysnatraemia common in hospitalised patients, but it is also independently associated with poor outcomes. In this study, the hyponatraemia group showed that $46.3 \%$ developed hyponatraemia on admission and $53.7 \%$ after 24 hours of hospitalisation, while $65.8 \%$ had persistent hyponatraemia. In the hypernatraemia group, patients were divided into community-acquired (50.4\%) and hospital-acquired (49.6\%) hypernatraemia, with $49.1 \%$ having persistent hypernatraemia. Mc Causland et al. ${ }^{[5]}$ also demonstrated a higher risk of mortality in orthopaedic surgical patients with abnormal preoperative serum sodium measurements (hazard ratio (HR) 2.47, 95\% confidence interval (CI) 1.33 - 4.59 for moderate/severe hyponatraemia; HR 1.80, 95\% CI $1.21-2.66$ for mild hyponatraemia; and HR $2.99,95 \%$ CI $1.79-4.98$ for hypernatraemia). ${ }^{[5]}$
Although a subanalysis of patient data from EuSOS found moderate to severe preoperative hypernatraemia to be associated with an increased risk of postoperative mortality, a statistically significant association between preoperative hyponatraemia and postoperative hyponatraemia was not demonstrated. ${ }^{[1]}$ This finding is in contrast to that reported in the studies by Leung et al. ${ }^{[2,3]}$ The two studies by Leung et al., ${ }^{[2,3]}$ which were analyses of a large, US surgical database, found that hyponatraemia and hypernatraemia were associated with a $44 \%$ higher risk of 30 -day postoperative mortality (odds ratio (OR) for hyponatraemia 1.44 (95\% CI 1.38 - 1.50); OR for hypernatraemia 1.44 (95\% CI 1.33 - 1.56)). The abovementioned studies also found preoperative hyponatraemia/hypernatraemia to be associated with a higher risk of postoperative cardiac complications, wound infection, pneumonia, extended postoperative length of stay and thromboembolism in patients undergoing major surgical procedures. This may suggest a potential geographical variation in the importance of preoperative sodium levels between US and European settings.

Klinck et al. ${ }^{[6]}$ propose a relationship between dysnatraemia and underlying neuroendocrine or inflammatory disorders. As neuroendocrine and inflammatory disorders are often related to the indication for surgery, or are present as comorbid conditions in surgical patients, these patients might be at risk of developing dysnatraemia. These authors investigated the clinical correlation 
of large perioperative sodium changes. A decrease in sodium concentration was associated with older patients ( $>60$ years) and those who had undergone procedures of longer duration. Those with diabetes or liver disease who were mechanically ventilated or receiving oxygen, and who used patient-controlled analgesia in the postoperative period, were more likely to have a large decrease in sodium than those who did not receive such analgesia. Major urological and renal procedures were also associated with this risk compared with a reference group comprising all inpatient laparoscopic operations. Obesity appeared to be protective against large decreases. ${ }^{[6}$

While there is literature describing the potential prognostic importance of preoperative serum sodium levels in overseas surgical populations, the importance of preoperative serum sodium levels in South African (SA) surgical patients is poorly described. ${ }^{[1-3,6]}$ As discordances in the importance of other preoperative clinical risk factors for inpatient mortality between SA and other surgical populations have been previously described, it is necessary to establish the clinical significance of abnormal preoperative serum sodium measurements on postoperative outcomes in SA surgical patients and not extrapolate its clinical significance from studies in other settings. ${ }^{[7]}$ This is made even more pertinent as there have already been differences identified in the clinical importance of hyponatraemia between US and European settings. Establishing the clinical importance of preoperative sodium levels in a SA setting would have implications as to whether these measurements should be included as a variable of interest during the future development of setting-specific perioperative risk stratification methods.

\section{Objective}

The objective of this study was to determine whether preoperative serum sodium measurements are associated with postoperative inpatient mortality in SA surgical patients.

\section{Methods}

\section{Study design and setting}

This was an unmatched case-control study of data collected as part of the South African Surgical Outcomes Study (SASOS). SASOS was a snapshot audit of surgical patients attending SA government hospitals during a 1-week period in $2014 .^{[8]}$

\section{Patients, data and sample size}

The SASOS database is comprised of 3927 SA patients $\geq 16$ years old, who underwent surgical procedures at 45 government hospitals between 19 and 26 May 2014. Various patient and clinical characteristics were collected during SASOS, including patient age, gender, comorbidities, preoperative laboratory test results (including serum sodium measurements), procedure data, and data related to postoperative inpatient mortality. Patients were classified as white and black, with black being inclusive of any patient who is of African, Indian or Coloured ethnicity. All laboratory tests were performed within 28 days before surgery. ${ }^{[8]}$

The definitions for hyponatraemia and hypernatraemia used in this study were adopted from the published literature (hyponatraemia: $<135 \mathrm{mEq} / \mathrm{L}$, hypernatraemia: $>144 \mathrm{mEq} / \mathrm{L}$, normal: 135 $144 \mathrm{mEq} / \mathrm{L}){ }^{[1-3]} \mathrm{A}$ case was defined as a patient who suffered inpatient mortality, while a control was defined as a patient who did not suffer inpatient mortality. Sample size calculations suggested that a study population of 513 patients (using a conventional 1:4 ratio of cases to controls in unmatched case-control studies (103 cases and 410 controls)) would be required for this research. Following exclusion of patients with missing data, cases and controls were randomly selected using a random number generator from the SASOS patient population until the specified sample size was attained. This was done to reduce selection bias, in accordance with published guidelines for the conduct of unmatched case-control studies. ${ }^{[9,10]}$

\section{Data analysis}

We followed published recommendations for the statistical analysis of unmatched case-control study data. ${ }^{[9,10]}$ To investigate the relationship between preoperative serum sodium and postoperative inpatient mortality, patient data were analysed using univariate/crude $\left(\chi^{2}\right.$ test, Fisher's exact test for categorical data or the Mann-Whitney test for continuous data) and multivariate/adjusted (unconditional logistic regression model) statistical methods. Results for the univariate statistical analysis are presented as frequencies with percentages, or medians with interquartile ranges (IQRs). Potential confounders identified from the univariate statistical analysis were accounted for in the unconditional logistic regression model. Results for the multivariate analysis are presented as ORs and $95 \%$ CIs. A $p$-value $<0.05$ was considered to be statistically significant. All statistical analyses were performed using SPSS version 24.0 (IBM Corp., USA).

\section{Ethical approval}

This study was approved by the Biomedical Research Ethics Committee of the University of KwaZulu-Natal, Durban, SA (ref. no. BE437/17)

\section{Results}

Table 1 shows the distribution of patient and clinical characteristics in the entire study population $(N=513)$, as well as the study population stratified into case and control groups ( $n=103$ and $n=410$, respectively). The median age of the study population was 46.0 (31.5 - 60.0) years; it comprised more males than females. Nine in every 10 patients were black. A third of patients had severe systemic disease (American Society of Anesthesiologists (ASA) score $>2$ ), while a quarter were current smokers. The prevalence of various comorbidities ranged between $0.6 \%$ and $56.3 \%$. Just over three-quarters of the study population had normal preoperative serum sodium measurements (77.8\%), with preoperative dysnatraemia present in the remaining $22.2 \%$ (18.5\% with hyponatraemia and $3.7 \%$ with hypernatraemia). Non-communicable disease pathology was the primary indication for surgery in almost half of the study population. Just over half of surgical procedures performed were urgent/emergent, with almost three-quarters undergoing major surgery. General anaesthesia was used in the majority of surgical procedures.

The univariate/crude statistical associations between various patient/clinical characteristics and postoperative inpatient mortality are shown in Table 1. Statistically significant associations with postoperative inpatient mortality were noted for age $(p<0.001)$, ASA score $>2(p<0.001)$, metastatic cancer $(p<0.001)$, HIV $(p=0.032)$, renal impairment $(p<0.001)$, preoperative anaemia $(p<0.001)$, preoperative serum sodium measurements $(p<0.001)$, urgent/emergent surgery $(p<0.001)$ and major surgery $(p<0.001)$. Specifically, the median age of the case group was higher than that of the control group (56.0 v. 43.0 years, respectively); a higher proportion of cases v. controls had an ASA score $>2(69.9 \%$ v. $23.9 \%)$, metastatic cancer $(11.7 \%$ v. $2.4 \%)$, renal impairment ( $14.6 \%$ v. $2.9 \%)$, urgent/emergent surgery $(76.9 \%$ v. $49.3 \%)$ and major surgery $(45.6 \%$ v. $22.4 \%)$. With regard to preoperative serum sodium measurements, the case group had a higher proportion of hyponatraemia and hypernatraemia compared with the control group ( $26.2 \%$ v. $16.5 \%$, and $10.7 \%$ v. $2.0 \%)$. 
Table 1. Distribution of patient/clinical characteristics in the study population and results of the univariate statistical analysis*

\begin{tabular}{|c|c|c|c|c|}
\hline Clinical characteristic & All patients $(N=513)$ & Cases $(n=103)$ & Controls $(n=410)$ & $p$-value \\
\hline Age, years (IQR) & $46.0(31.5-60.0)$ & $56.0(39.0-65.0)$ & $43.0(30.0-58.0)$ & $<0.001$ \\
\hline Gender & & & & 0.793 \\
\hline Male & $273(53.2)$ & $56(54.4)$ & $217(52.9)$ & \\
\hline Female & $240(46.8)$ & $47(45.6)$ & $193(47.1)$ & \\
\hline Race & & & & 0.733 \\
\hline White & $55(10.7)$ & $12(11.7)$ & $43(10.5)$ & \\
\hline Black & $458(89.3)$ & $91(88.3)$ & $367(89.5)$ & \\
\hline ASA score $>2$ & & & & $<0.001$ \\
\hline Yes & $170(33.1)$ & $72(69.9)$ & $98(23.9)$ & \\
\hline No & $343(66.9)$ & $31(30.1)$ & $312(76.1)$ & \\
\hline Current smoker & & & & 0.201 \\
\hline Yes & $135(26.3)$ & $22(21.4)$ & $113(27.6)$ & \\
\hline No & $378(73.7)$ & $81(78.6)$ & $297(72.4)$ & \\
\hline Cardiovascular disease & & & & 0.438 \\
\hline Yes & $54(10.5)$ & $13(12.6)$ & $41(10.0)$ & \\
\hline No & $459(89.5)$ & $90(87.4)$ & $369(90.0)$ & \\
\hline Metastatic cancer & & & & $<0.001$ \\
\hline Yes & $22(4.3)$ & $12(11.7)$ & $10(2.4)$ & \\
\hline No & $491(95.7)$ & $91(88.3)$ & $400(97.6)$ & \\
\hline Cirrhosis & & & & 0.490 \\
\hline Yes & $3(0.6)$ & $1(1.0)$ & $2(0.5)$ & \\
\hline No & $510(99.4)$ & $102(99.0)$ & $408(99.5)$ & \\
\hline Chronic obstructive pulmonary disease & & & & 0.808 \\
\hline Yes & $37(7.2)$ & $8(7.8)$ & $29(7.1)$ & \\
\hline No & $476(92.8)$ & $95(92.2)$ & $381(92.9)$ & \\
\hline HIV & & & & 0.032 \\
\hline Yes & $80(15.6)$ & $9(8.7)$ & $71(17.3)$ & \\
\hline No & $433(84.4)$ & $94(91.3)$ & $339(82.7)$ & \\
\hline Diabetes & & & & 0.277 \\
\hline Yes & $68(13.3)$ & $17(16.5)$ & $51(12.4)$ & \\
\hline No & $445(86.7)$ & $86(83.5)$ & $359(87.6)$ & \\
\hline Renal impairment & & & & $<0.001$ \\
\hline Yes & $27(5.3)$ & $15(14.6)$ & $12(2.9)$ & \\
\hline No & $486(94.7)$ & $88(85.4)$ & $398(97.1)$ & \\
\hline Preoperative anaemia & & & & $<0.001$ \\
\hline Yes & $262(51.1)$ & $74(71.8)$ & $188(45.9)$ & \\
\hline No & $251(48.9)$ & $29(28.2)$ & $222(54.1)$ & \\
\hline Other comorbidity not otherwise specified ${ }^{\ddagger}$ & & & & 0.181 \\
\hline Yes & $289(56.3)$ & $52(50.5)$ & $237(57.8)$ & \\
\hline No & $224(43.7)$ & $51(49.5)$ & $173(42.2)$ & \\
\hline Preoperative sodium measurement & & & & $<0.001$ \\
\hline Hyponatraemia & $95(18.5)$ & $27(26.2)$ & $68(16.5)$ & \\
\hline Hypernatraemia & $19(3.7)$ & $11(10.7)$ & $8(2.0)$ & \\
\hline Normal & $399(77.8)$ & $65(63.1)$ & $334(81.5)$ & \\
\hline Indication for surgery & & & & 0.302 \\
\hline Non-communicable disease & $240(46.8)$ & $43(41.7)$ & $197(48.0)$ & \\
\hline Injury/trauma & $161(31.4)$ & $32(31.1)$ & $129(31.5)$ & \\
\hline Infectious disease & $112(21.8)$ & $28(27.2)$ & $84(20.5)$ & \\
\hline Urgent/emergent surgery & & & & $<0.001$ \\
\hline Yes & $284(55.4)$ & $82(79.6)$ & $202(49.3)$ & \\
\hline No & $229(44.6)$ & $21(20.4)$ & $208(50.7)$ & \\
\hline Major surgery & & & & $<0.001$ \\
\hline Yes & $139(27.1)$ & $47(45.6)$ & $92(22.4)$ & \\
\hline No & $374(72.9)$ & $56(54.4)$ & $318(77.6)$ & \\
\hline Surgery with general anaesthesia & & & & 0.093 \\
\hline Yes & $364(71.0)$ & $80(77.7)$ & $284(69.3)$ & \\
\hline No & $149(29.0)$ & $23(22.3)$ & $126(30.7)$ & \\
\hline
\end{tabular}




\begin{tabular}{|c|c|c|}
\hline Clinical characteristic & OR $(95 \% \mathrm{CI})$ & $p$-value ${ }^{*}$ \\
\hline Age (per year increase) & $1.04(1.02-1.06)$ & $<0.001$ \\
\hline \multicolumn{3}{|l|}{ Gender } \\
\hline Male & $0.83(0.46-1.51)$ & 0.541 \\
\hline Female & Reference & - \\
\hline \multicolumn{3}{|l|}{ Race } \\
\hline White & $0.44(0.18-1.10)$ & 0.078 \\
\hline Black & Reference & - \\
\hline \multicolumn{3}{|l|}{ ASA score $>2$} \\
\hline Yes & $5.00(2.64-9.45)$ & $<0.001$ \\
\hline No & Reference & - \\
\hline \multicolumn{3}{|l|}{ Current smoker } \\
\hline Yes & $0.89(0.45-1.75)$ & 0.730 \\
\hline No & Reference & - \\
\hline \multicolumn{3}{|l|}{ Cardiovascular disease } \\
\hline Yes & $0.34(0.10-1.14)$ & 0.079 \\
\hline No & Reference & - \\
\hline \multicolumn{3}{|l|}{ Metastatic cancer } \\
\hline Yes & $2.13(0.53-8.58)$ & 0.288 \\
\hline No & Reference & - \\
\hline \multicolumn{3}{|l|}{ Cirrhosis } \\
\hline Yes & $0.99(0.05-18.16)$ & 0.993 \\
\hline No & Reference & - \\
\hline \multicolumn{3}{|c|}{ Chronic obstructive pulmonary disease } \\
\hline Yes & $0.51(0.14-1.80)$ & 0.292 \\
\hline No & Reference & - \\
\hline \multicolumn{3}{|l|}{ HIV } \\
\hline Yes & $0.31(0.07-1.30)$ & 0.109 \\
\hline No & Reference & - \\
\hline \multicolumn{3}{|l|}{ Diabetes } \\
\hline Yes & $0.47(0.13-1.66)$ & 0.242 \\
\hline No & Reference & - \\
\hline \multicolumn{3}{|l|}{ Renal impairment } \\
\hline Yes & $3.22(1.14-9.08)$ & 0.027 \\
\hline No & Reference & - \\
\hline \multicolumn{3}{|l|}{ Preoperative anaemia } \\
\hline Yes & $1.72(0.94-3.14)$ & 0.078 \\
\hline No & Reference & - \\
\hline \multicolumn{3}{|c|}{ Other comorbidity not otherwise specified ${ }^{\dagger}$} \\
\hline Yes & $0.83(0.22-3.06)$ & 0.777 \\
\hline No & Reference & - \\
\hline \multicolumn{3}{|l|}{ Preoperative sodium measurement } \\
\hline Hyponatraemia & $1.39(0.70-2.76)$ & 0.346 \\
\hline Hypernatraemia & $4.21(1.19-14.83)$ & 0.025 \\
\hline Normal & Reference & - \\
\hline \multicolumn{3}{|l|}{ Indication for surgery } \\
\hline Non-communicable disease & $0.99(0.47-2.09)$ & 0.976 \\
\hline Injury/trauma & $1.35(0.61-2.98)$ & 0.458 \\
\hline Infectious disease & Reference & - \\
\hline \multicolumn{3}{|l|}{ Urgent/emergent surgery } \\
\hline Yes & $3.57(1.88-6.80)$ & $<0.001$ \\
\hline No & Reference & - \\
\hline \multicolumn{3}{|l|}{ Major surgery } \\
\hline Yes & $2.69(1.42-5.06)$ & 0.002 \\
\hline No & Reference & - \\
\hline \multicolumn{3}{|l|}{ Surgery with general anaesthesia } \\
\hline Yes & $2.11(1.03-4.33)$ & 0.041 \\
\hline No & Reference & - \\
\hline
\end{tabular}


The results of the multivariate/adjusted statistical analysis are shown in Table 2. Patients with preoperative hypernatraemia, but not preoperative hyponatraemia, were found to have a higher risk of postoperative inpatient mortality compared with those who had normal preoperative serum sodium measurements (OR 4.21, 95\% CI 1.19 - 14.83, $p=0.025$ for hypernatraemia; OR $1.39,95 \%$ CI $0.70 .0-2.76$, $p=0.346$ for hyponatraemia). Other patient/clinical characteristics independently associated with a higher risk of postoperative inpatient mortality were: increasing age (OR 1.04, 95\% CI 1.02 - 1.06, $p<0.001$ ), ASA score $>2$ (OR 5.00, 95\% CI 2.64 - 9.45, $p<0.001$ ), renal impairment (OR 3.22, 95\% CI $1.14-9.08, p=0.027$ ), urgent/emergent surgery (OR 3.57, 95\% CI $1.88-6.80, p<0.001$ ), major surgery (OR 2.69, 95\% CI $1.42-5.06, p=0.002$ ), and general anaesthesia (OR 2.11, 95\% CI $1.03-4.33, p=0.041$ ).

\section{Discussion}

The crude statistical association reported between preoperative serum sodium measurements and postoperative inpatient mortality in our univariate statistical analysis translated into a statistically significant, independent association when potential confounders were accounted for. Specifically, preoperative hypernatraemia was associated with a four-fold higher risk of postoperative inpatient mortality compared with preoperative serum sodium measurements within the normal range. Preoperative hyponatraemia was not independently associated with postoperative inpatient mortality compared with preoperative serum sodium measurements within the normal range. In a subanalysis of data from EuSOS, preoperative hypernatraemia was independently associated with a 1.3 - 3.4-fold higher risk of postoperative mortality, while preoperative hyponatraemia was not identified as a risk factor for postoperative inpatient mortality. ${ }^{[1]}$ In the two studies by Leung et al., ${ }^{[2,3]}$ patients with preoperative hyponatraemia or preoperative hypernatraemia had a $44 \%$ higher risk of postoperative mortality compared with those with normal preoperative serum sodium measurements.

The similarity of our study findings to those of a European cohort (EuSOS) and one of the US studies by Leung et al..$^{[2]}$ suggests that preoperative hypernatraemia is a potentially important prognostic variable in both lower/middle-income and higher-income countries. While we did not observe increased mortality in patients with preoperative hyponatraemia, which was reported in one of the studies by Leung et al. ${ }^{[3]}$ our finding for hyponatraemia is similar to that reported in the EuSOS sub-study. Cecconi et al. ${ }^{[1]}$ provided a possible explanation for the discordant findings between their subanalysis of the EuSOS data and the study of Leung et al., ${ }^{[2]}$ suggesting that the EuSOS prospective design and extensive data collection during the study period had allowed them better control for potential confounders in their multivariate analysis than was possible in the study of Leung et al. As the design and conduct of SASOS were based on those of EuSOS, it is likely that the explanation for the discordancy in study findings for hyponatraemia between our and Leung et al's studies is the same as that proposed by Cecconi et al. ${ }^{[1]}$

Our study findings, as well as the relatively cheap cost and availability of serum sodium measurements performed at diagnostic laboratories or as point-of-care tests, raise interest in the possibility of using preoperative serum sodium measurements for prognostic purposes in resource-limited settings. Further research investigating the prognostic accuracy of preoperative serum sodium measurements in resource-limited settings is recommended.

While we present findings related to other patient/clinical characteristics, we advise caution when interpreting these findings, as our unmatched case-control study was specifically designed to determine the relationship between preoperative serum sodium measurements and postoperative inpatient mortality. The other patient/clinical characteristics were included in the multivariate statistical analysis as a method to control for potential confounders, according to recommendations for unmatched case-control studies. ${ }^{[9,10]}$ A broad investigation of the potential impact of these patient/clinical characteristics on postoperative inpatient mortality has already been described as a component of the main SASOS publication, and more specific investigations would require additional case-control studies to be performed. ${ }^{[8]}$

\section{Study strengths and limitations}

A strength of our study is that we present a subanalysis of prospectively collected data from a large, representative study of perioperative outcomes in SA surgical patients. An important limitation of our study is that there might be some potentially relevant patient/clinical characteristics (such as medication use) that were not recorded during the data collection phase of SASOS. Therefore, we could not control for any confounding attributed to these characteristics in our multivariate statistical analysis. The cause of inpatient mortality was also not recorded as part of SASOS, and therefore all-cause inpatient mortality is reported in our study. As the SASOS design was based on that of EuSOS, we also acknowledge that our study has additional limitations similar to those reported by Cecconi et al. ${ }^{[1]}$ Further prospective research is required to confirm our findings and address the limitations of our study.

\section{Conclusions}

Preoperative hypernatraemia was found to be an important risk factor for postoperative mortality in SA surgical patients. Hyponatraemia was not found to be a risk factor for postoperative mortality in our study. Further research is required to assess the prognostic accuracy of preoperative serum sodium measurements in SA surgical patients, as well as to address the limitations of our study.

\section{Acknowledgements. None.}

Author contributions. MR: conceptualisation of research, interpretation of results and manuscript preparation; YM: conceptualisation of research, statistical analysis, interpretation of results and manuscript preparation; and PDG: critical evaluation of draft manuscript.

Funding. None.

Conflicts of interest. None.

1. Cecconi M, Hochrieser H, Chew M, et al. Preoperative abnormalities in serum sodium concentration are associated with higher in-hospital mortality in patients undergoing major surgery. Br J Anaesth 2016;116(1):63-69. https://doi.org/10.1093/bja/aev373

2. Leung AA, McAlister FA, Finlayson SR, Bates DW. Preoperative hypernatraemia predicts increased perioperative morbidity and mortality. Am J Med 2013;126(10):877-886. https://doi.org/10.1016/j. perioperative morbid
amjmed.2013.02.039

3. Leung AA, McAlister FA, Rogers SO, Jr., Pazo V, Wright A, Bates DW. Preoperative hyponatraemia and perioperative complications. Arch Intern Med 2012;172(19):1474-1481. https://doi.org/10.1001/ archinternmed.2012.3992

4. Hu J, Wang Y, Geng X, et al. Dysnatraemia is an independent indicator of mortality in hospitalized patients. Med Sci Monitor 2017;23:2408-2425. https://doi.org/10.12659/msm.902032

5. Mc Causland FR, Wright J, Waikar SS. Association of serum sodium with morbidity and mortality in hospitalized patients undergoing major orthopedic surgery. J Hosp Med 2014;9(5):297-302. https:// doi.org/10.1002/jhm.2168

6. Klinck J, McNeill L, Di Angelantonio E, Menon DK. Predictors and outcome impact of perioperative serum sodium changes in a high-risk population. Br J Anaesth 2015;114(4):615-622. https://doi. org/10.1093/bja/aeu409

7. Moodley Y, Biccard BM. Predictors of in-hospital mortality following non-cardiac surgery: Findings from an analysis of a South African hospital administrative database. S Afr Med J 2015;105(2):126-129. https://doi.org/10.7196/SAMJ.826

8. Biccard BM, Madiba TE, South African Surgical Outcomes Study I. The South African Surgical Outcomes Study: A 7-day prospective observational cohort study. S Afr Med J 2015;105(6):465-475. https://doi.org10.7196/SAMJ.9435

9. Breslow NE, Day NE. Statistical methods in cancer research. IARC Workshop 25 - 27 May 1983. IARC Sci Publ 1987;(82):1-406.

10. Mandrekar JN, Mandrekar SJ. Case-control study design: What, when, and why? J Thorac Oncol 2008;3(12):1371-1372. https://doi.org/10.1097/JTO.0b013e31818dd97b

Accepted 12 April 2018. 